\title{
Child deaths at National District Hospital, Free State: one a month is better than one a week
}

\author{
H Brits $^{\text {a* iD }}$ \\ ${ }^{a}$ Faculty of Health Sciences, Department of Family Medicine, University of the Free State, Bloemfontein, South Africa \\ *Email:britsh@ufs.ac.za
}

Background: The United Nations set a two-thirds reduction in child mortality between 1990 and 2015 in the Millennium Development Goals (MDGs) of 2000. The National Department of Health (NDoH) introduced strategies to achieve these MDGs, which included new vaccines, better HIV care and training of healthcare workers. This study investigated whether the strategies implemented by the NDoH decreased child mortality (MDG 4) at National District Hospital (NDH).

Method: A retrospective file review was done on all children that died in NDH from 2008 to 2015. Data were collected from patient files and ChildPIP data forms. Deaths before and after the implementation of the strategies were compared.

Results: A total of 209 children died during the study period. The mortality rate decreased from 47 per thousand admissions and stabilised at 15 per thousand admissions for the past five years. Deaths due to acute gastroenteritis decreased from $67 \%$ of the total to less than 40\%. Pneumonia as the main cause of death decreased from 44 during the 2008-2010 period to 19 during the 2011-2015 period. More than $90 \%$ of the children who died were malnourished. There was no statistically significant improvement in the malnutrition rates during the study periods $(p=0.85)$.

Conclusion: Child deaths decreased from one a week to one a month at NDH. Strategies to meet the MDG 4 targets, like the introduction of the Rotavirus and Pneumococcal vaccine, the scale-up of anti-retroviral treatment and Prevention of Mother to Child transmission of HIV and better Integrated Management of Childhood Illness training all contributed to the better outcome.

Keywords: child deaths, gastroenteritis, health strategies, HIV, malnutrition, Millennium Development Goal 4

\section{Introduction}

At the 2000 Millennium Summit of the United Nations (UN), eight Millennium Development Goals (MDGs) were adopted. All UN member states at that time, as well as other international organisations, committed to supporting and helping to achieve the MDGs by $2015 .{ }^{1}$ The aim of MDG 4 is to reduce the mortality rate of children under five years by two-thirds during the period 1990 to 2015 . For the purpose of this study, two goals are closely linked to MDG4: MDG 1, which addresses extreme hunger and poverty, and MDG 6, which addresses human immunodeficiency virus/acquired immunodeficiency syndrome (HIV/AIDS) and other diseases.'

The National Department of Health (NDoH) in South Africa introduced some strategies from 2008 onwards specifically to address MDG 4. The quality and integrity of data was overseen by appointing the Health Data Advisory and Co-ordination Committee (HDACC), which set criteria for data to be used for specific indicators. ${ }^{2}$ The most important initiatives introduced by the $\mathrm{NDoH}$ included the prevention of mother-to-child transmission (PMTCT) of HIV programme, improved paediatric HIV treatment programmes, immunisations against rotavirus and invasive pneumococcal disease (implemented in 2008), upscaling of the use of vitamin $A$, and intensifying the management of children with severe malnutrition and other sick children with the Integrated Management of Childhood IIIness programme (IMCl). ${ }^{3}$

A study conducted between 2007 and 2008 at National District Hospital (NDH) in Bloemfontein, Free State found that $5 \%$ (49/983) of admitted children died, $67 \%$ of them due to gastroenteritis. Underlying poor nutritional status and/or HIVpositive status were present in $96 \%$ of deaths. ${ }^{4}$

\section{Aim of the study}

The aim of this study was to investigate whether the strategies implemented by the NDoH to decrease child mortality (MDG 4) were evident in child deaths at NDH.

\section{Method}

\section{Study design}

This was a retrospective file review. The study population and sample included all children who died in NDH's paediatric ward between January 2008 and December 2015.

National District Hospital is a 196-bed district hospital situated between a secondary and tertiary hospital in Bloemfontein. The paediatric ward has 23 beds and a paediatrician does weekly training rounds in the hospital. Protocols and guidelines from the $\mathrm{NDoH}$ are followed in the hospital. During the study period, no notable changes occurred regarding the availability of beds or referral protocols. The doctor in charge of the ward and the visiting paediatrician also remained the same during the study period. All relevant new $\mathrm{NDoH}$ protocols were implemented during the study period.

\section{Data collection}

For each death, demographic data, cause of death and underlying conditions were obtained from hospital files and Child Healthcare Problem Identification Programme (Child PIP) data, and recorded on a data form. A comparison between deaths before the implementation of the MDG strategies and after the implementation was done by dividing the deaths into two groups, namely 2008-2010 and 2011-2015. The majority of strategies from NDoH were implemented between 2008 and 2010 and therefore 
those years were grouped together. The longer period afterwards was chosen to look for sustainability and not short-term changes.

\section{Pilot study}

No pilot study was done as the data form had already been used in a study to investigate child deaths at NDH for a 12-month period during 2007-2008. ${ }^{4}$

\section{Data analysis}

The Department of Biostatistics of the Faculty of Health Sciences, University of the Free State (UFS) assisted with data analysis using SAS ${ }^{\circledR}$ version 9.3 (SAS Institute, Cary, NC, USA). Results are summarised by frequencies, tables, graphs, percentages and means. $P$-values $<0.05$ were considered statistically significant.

\section{Ethical aspects}

The original study as well as the continuation of the study was approved by the Ethics Committee of the Faculty of Health Science, UFS, and the Free State Department of Health.

\section{Results}

A total of 211 deaths occurred during the 8-year period from 2008 to 2015 . Slightly more female patients $(53 \%)$ died; however, there was no significant difference for gender $(p=0.85)$. The age distribution at time of death was between 1 month and 192 months, with a median age of 12 months. Seven of the children who died were older than 13 years and male. The majority of children died within two days of admission. The duration from admission to death varied between minutes and 277 days, with a standard deviation of 23.2 days.

The number of admissions decreased from 1000 per year (20082009 ) to 850 per year (2012-2015). In Figure 1, the number of deaths is displayed per year.

The mortality rate decreased from 47 per 1000 admissions (2008-2010) and stabilised at 15 per 1000 admissions over the past 5 years (2011-2015).

Acute gastroenteritis was the main cause of death during the study period. Deaths due to acute gastroenteritis decreased from $67 \%$ of the total to less than $40 \%$ of the total for the past 8 years. Total admissions to hospital due to acute gastroenteritis decreased by $25 \%$. Some $40 \%$ of the children who died from acute gastroenteritis were form rural areas, and the majority of these children died on or shortly after arrival.

Pneumonia as the main cause of death decreased from 44 cases during the 2008-2010 period to 19 cases during the 2011-2015 period. This decrease is statistically significant $(p=0.024)$. The decrease for bacterial pneumonia between the two study periods is $55 \%$. Deaths due to HIV complications, such as pneumocystis pneumonia (PCP), last occurred in 2010. The number of pneumonia admissions stayed constant over the total study period. The number of pneumonia deaths is displayed per year in Figure 2.

The HIV-positive rates of the children who died decreased from $86 \%(2008-2010)$ and stabilised at 64\% (2011-2015). This is statistically significant $(p=0.016)$. All 28 of the children 8 years and older who died were HIV-positive and were admitted for palliative/terminal care.

More than $90 \%$ of the children who died were malnourished. There was no statistical significant improvement in the

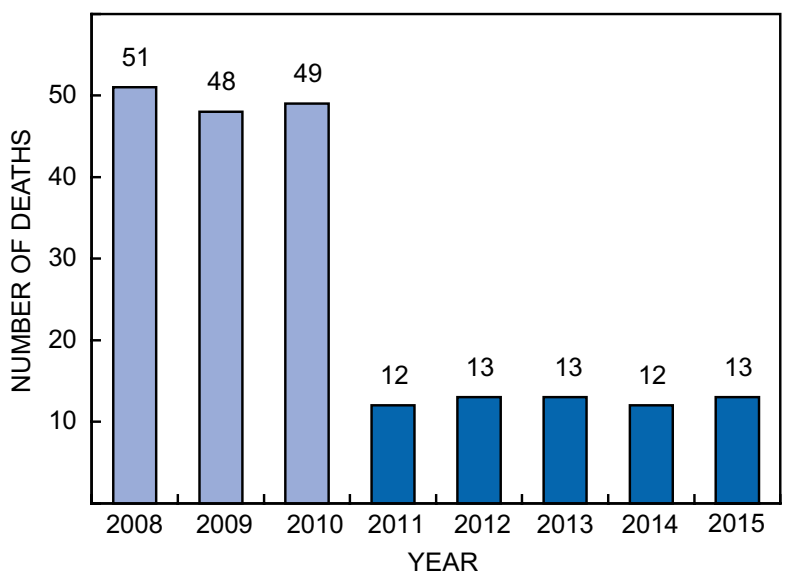

Figure 1: Number of deaths per year during the overall study period.

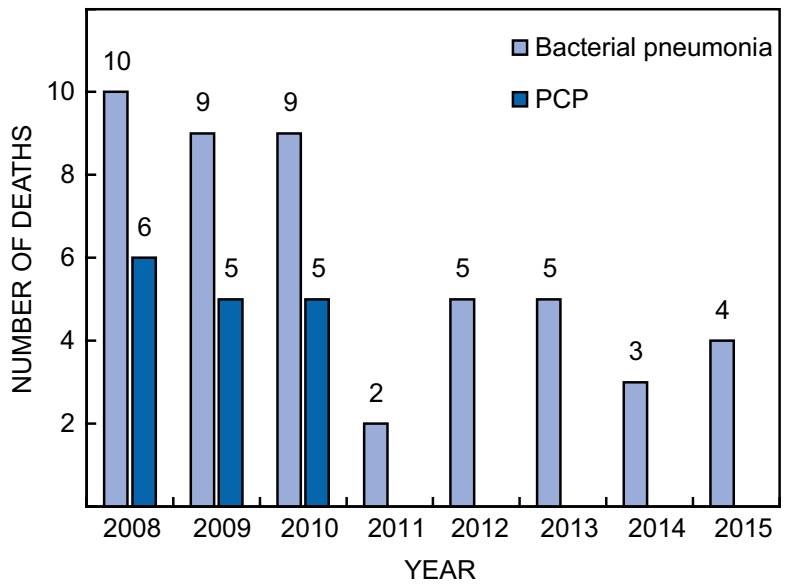

Figure 2: Number of pneumonia deaths due to bacterial pneumonia and pneumocystis pneumonia (PCP) during the overall study period.

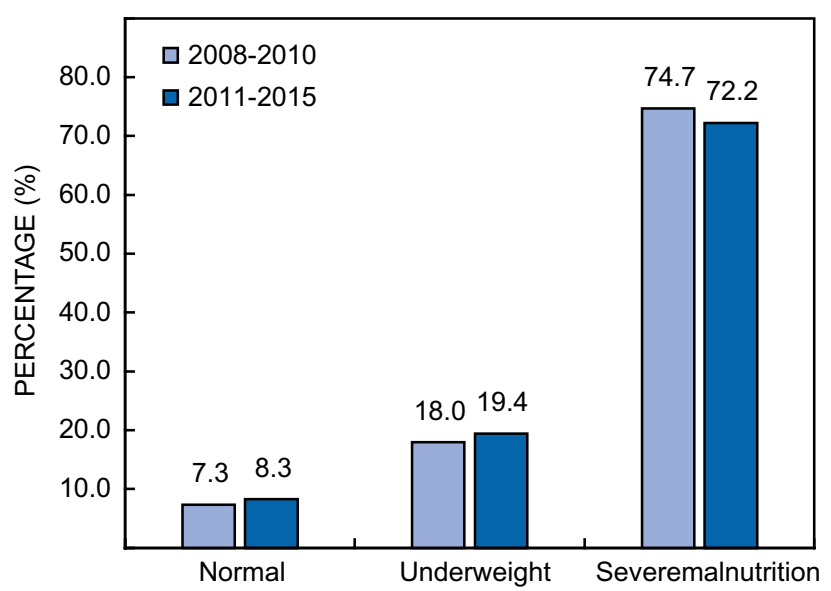

Figure 3: Nutritional status in the two study periods.

malnutrition rates during the study periods $(p=0.85)$. In Figure 3 , the nutritional status of the children who died is displayed.

\section{Discussion}

The number of child deaths in NDH's paediatric ward as well as the mortality rate of admissions decreased from the 2008-2010 period and stabilised over the 2011-2015 period. Data from 
Unicef, the World Bank and the 2014 Committee on Morbidity and Mortality (CoMMiC) in children under five years report all confirmed a decrease in the under-five mortality rate (U5MR) in South Africa. ${ }^{5-7}$ The U5MR in South Africa decreased from $58.4 / 1000$ in 2007 to $38.5 / 1000$ in 2011. For the Free State, the U5MR decreased from 34.9/1 000 in 2009 to 22.3/1 000 in 2012, and for the Mangaung area (where NDH is situated) it decreased from 32.6/1 000 in 2009 to 17.8/1 000 in 2012. ${ }^{7}$

The gender distribution of the deaths stayed the same for the study period with more female patients $(53 \%)$ who died. Data for South Africa and the Free State, however, show a male dominance of 52.2\%. ${ }^{8}$ As in the 2007-2008 study, many of the children died on or shortly after presentation to $\mathrm{NDH}$. The situation regarding non-availability of 24-hour medical services did not improve during the study period. ${ }^{4}$

Acute gastroenteritis was the main cause of death during the study period, as is the case for the rest of South Africa. The decrease in gastroenteritis cases as well as related deaths may be attributed to the introduction of the rotavirus vaccine in August 2009. According to a study published in 2014, the rotavirus vaccine gave $73 \%$ protection against rotavirus-associated severe diarrhoeal disease in South Africa. ${ }^{9}$ This protection was also demonstrated in studies in Australia and Mexico. ${ }^{10}$ The seconddose rotavirus vaccine coverage increased from 1.8\% in 2009 to $100.8 \%$ in 2012 in the Free State. ${ }^{9}$

Pneumonia as a major cause of death stayed in second place but the decrease in deaths was statistically significant. According to the Child PIP report, the pneumonia deaths in under-fives decreased by 53\% between 2008 and $2012 .{ }^{6}$ This compares well with the decrease of $55 \%$ in bacterial pneumonia deaths found in our study. The introduction of the pneumococcal vaccine (PCV) in 2009 could be a contributing factor to this decrease. The national coverage of PCV in nine-monthold children increased from $10.4 \%$ in 2010 to $99 \%$ in 2012 . In the USA, a $36 \%$ decrease in under two-year-old pneumonia admissions occurred three to four years after the introduction of the PCV. ${ }^{11}$

The inclusive and more assessable (distance learning and computerbased) Integrated Management of Childhood IIIness (IMCI) programme, as a strategy to decrease child mortality according to MDG 4, may also be a contributing factor to the decrease in child deaths as more trained nurses are available to initiate treatment in severely ill children with pneumonia and gastroenteritis. ${ }^{12}$

The statistically significant reduction in the percentage of HIVpositive children who died can be attributed to the extensive PMTCT and HIV treatment guidelines for both children and adults. ${ }^{13}$ A Health Systems Research Council publication stated that child mortality was reduced by $20 \%$ due to the scale-up of antiretroviral (ARV) treatment. ${ }^{14}$

A major concern is that malnutrition did not improve over the eightyear study period, despite the fact that the South African government introduced numerous initiatives to improve the nutritional status of children. These initiatives include child support grants, care dependency grants and feeding schemes at schools. According to the 2014 CoMMiC report, the Free State was the only province where the incidence of severe malnutrition increased during the 20092013 period. ${ }^{6}$ The national target was set at $1 \%$ while the figure for the Free State increased from 3.9\% in 2009 to $10.7 \%$ in $2013 .{ }^{6}$

\section{Conclusion}

During the study period, there was a decrease from one child death per week (2008-2010) to one child death per month (2011-2015) for children admitted to the paediatric ward at NDH. Strategies to meet the MDG 4 targets, such as the introduction of the rotavirus and pneumococcal vaccine, the scale-up of ARVs and PMTCT treatment programmes, and better $I \mathrm{MCl}$ training all contributed to the better outcome. Effective HIV treatment and prevention programmes (MDG 6) played a major role in the decrease in child mortality at NDH. However, the increase in malnutrition in the Free State is of great concern, and strategies to assess and improve the feeding schemes should be implemented urgently.

Acknowledgements - The authors wish to thank Ms T. Mulder, medical editor, School of Medicine, UFS, for technical and editorial preparation of the manuscript.

Disclosure of interest - The author reports no conflicts of interest.

\section{ORCID}

H Brits (D) http://orcid.org/0000-0002-5183-5345

\section{References}

1. United Nations. We can end poverty. The Millennium Development Goals. 2015 [cited 2016 Dec]. Available from: http://www.un.org/en/ mdg/summit2010/pdf/List\%20of\%20MDGs\%20English.pdf

2. Bamford L. Maternal, newborn and child health. South African Health Review 2012/2013. Durban: Health Systems Trust; 2013 [cited 2016 Dec]. Available from: http://www.hst.org.za/sites/default/files/ Chapter4_Maternal,\%20Newborn_and\%20Child_Health.pdf

3. Statistics South Africa. Millennium Development Goals. Goal 4. Reduce child mortality. 2010 [cited 2016 Dec]. Available from: http:// www.statssa.gov.za/MDG/2010_MDG_GOAL_4_REDUCE_CHILD_ MORTALITY.pdf

4. Brits $H$, Joubert $G$. An investigation into the dramatic increase in deaths from gastroenteritis during the summer of 2007/08 at National District Hospital, Bloemfontein, Free State. SA Fam Pract. 2009;51(5):418-22.

5. The World Bank. Reduce child mortality by 2015. 2016 [cited 2016 Dec] Available from: http://www.worldbank.org/mdgs/child_mortality.html

6. Kwazulu-Natal Department of Health. 2nd Triennial Report of the Committee on Morbidity and Mortality in Children under 5 years (COMMiC). 2014 [cited Dec 2016]. Available from: http://www. kznhealth.gov.za/mcwh/2nd-CoMMiC-Triennial-Report-2014.pdf

7. Unicef. Child Mortality. Unicef Statistics Jun 2016. [cited Dec 2016]. Available from http://data.unicef.org/child-mortality/under-five.html

8. De Lannoy A, Swartz S, Lake L, et al., editors. South African Child Gauge 2015. Cape Town: Children's Institute, University of Cape Town; 2015.

9. Madhi SA, Bamford L, Ngcobo N. Effectiveness of pneumococcal conjugate vaccine and rotavirus vaccine introduction into the South African public immunisation programme. S Afr Med J. 2014;104(3 Suppl 1):228-34. doi:10.7196/SAMJ.7597.

10. Gastañaduy PA, Sánchez-Uribe E, Esparza-Aguilar M, et al. Effect of rotavirus vaccine on diarrhea mortality in different socioeconomic regions of Mexico. Pediatrics. 2013;131(4):e1115-20. doi:10.1542/peds.2012-2797.

11. Griffin MR, Zhu Y, Moore MR, et al. U.S. hospitalizations for pneumonia after a decade of pneumococcal vaccination. $N$ Engl J Med. 2013;369(2):155-63. doi:10.1056/NEJMoa1209165.

12. Were W. Pre-service and in-service capacity building: lessons learned from Integrated Management of Childhood IIIness (IMCI). Switzerland: World Health Organization; 2014.

13. UNAIDS. South Africa HIV epidemic profile. 2014 [cited 2016 Dec]. Available from: http://www.unaidsrstesa.org/wp-content/ uploads/2015/05/UNAids-Profile-South-Africa.pdf-18-Feb.pdf

14. Shisana O, Rehle T, Simbayi LC, et al. South African National HIV Prevalence, Incidence and Behaviour Survey, 2012. Cape Town: HSRC Press; 2014. 